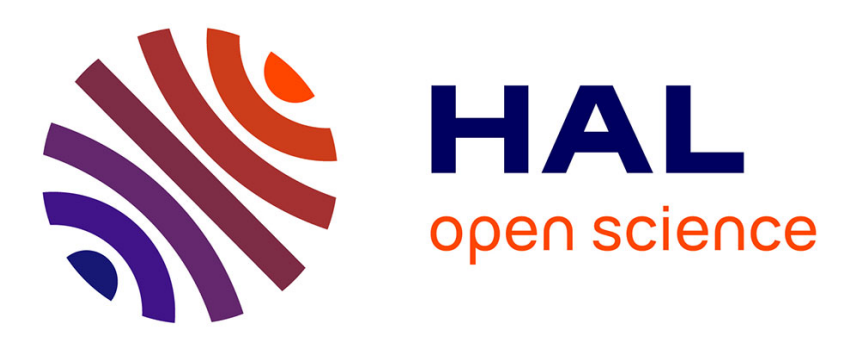

\title{
An explicit formula for the intersection of two polynomials of regular languages \\ Jean-Eric Pin
}

\section{To cite this version:}

Jean-Eric Pin. An explicit formula for the intersection of two polynomials of regular languages. Developments in Language Theory, - 17th International Conference, DLT 2013, Jun 2013, Champs sur Marne, France. pp.31-45. hal-01247099

\section{HAL Id: hal-01247099 \\ https://hal.science/hal-01247099}

Submitted on 21 Dec 2015

HAL is a multi-disciplinary open access archive for the deposit and dissemination of scientific research documents, whether they are published or not. The documents may come from teaching and research institutions in France or abroad, or from public or private research centers.
L'archive ouverte pluridisciplinaire HAL, est destinée au dépôt et à la diffusion de documents scientifiques de niveau recherche, publiés ou non, émanant des établissements d'enseignement et de recherche français ou étrangers, des laboratoires publics ou privés. 


\title{
An explicit formula for the intersection of two polynomials of regular languages
}

\author{
Jean-Éric Pin ${ }^{1 \star}$ \\ LIAFA, University Paris-Diderot and CNRS, France.
}

\begin{abstract}
Let $\mathcal{L}$ be a set of regular languages of $A^{*}$. An $\mathcal{L}$-polynomial is a finite union of products of the form $L_{0} a_{1} L_{1} \cdots a_{n} L_{n}$, where each $a_{i}$ is a letter of $A$ and each $L_{i}$ is a language of $\mathcal{L}$. We give an explicit formula for computing the intersection of two $\mathcal{L}$-polynomials. Contrary to Arfi's formula (1991) for the same purpose, our formula does not use complementation and only requires union, intersection and quotients. Our result also implies that if $\mathcal{L}$ is closed under union, intersection and quotient, then its polynomial closure, its unambiguous polynomial closure and its left [right] deterministic polynomial closure are closed under the same operations.
\end{abstract}

\section{Introduction}

Let $\mathcal{L}$ be a set of regular languages of $A^{*}$. An $\mathcal{L}$-polynomial is a finite union of products of the form $L_{0} a_{1} L_{1} \cdots a_{n} L_{n}$, where each $a_{i}$ is a letter of $A$ and each $L_{i}$ is a language of $\mathcal{L}$. The polynomial closure of $\mathcal{L}$, denoted by $\operatorname{Pol}(\mathcal{L})$, is the set of all $\mathcal{L}$-polynomials.

It was proved by Arfi 1 that if $\mathcal{L}$ is closed under Boolean operations and quotient, then $\operatorname{Pol}(\mathcal{L})$ is closed under intersection. This result was obtained by giving an explicit formula for computing the intersection of two polynomials of regular languages.

It follows from the main theorem of $[\underline{6}$ that Arfi's result can be extended to the case where $\mathcal{L}$ is only closed under union, intersection and quotient. However, this stronger statement is obtained as a consequence of a sophisticated result involving profinite equations and it is natural to ask for a more elementary proof.

The objective of this paper is to give a new explicit formula for computing the intersection of two $\mathcal{L}$-polynomials. Contrary to the formula given in [1, our formula only requires using union, intersection and quotients of languages of $\mathcal{L}$. Our proof is mainly combinatorial, but relies heavily on the notion of syntactic ordered monoid, a notion first introduced by Schützenberger [14] (see also [10]). The main difficulty lies in finding appropriate notation to state the formula, but then its proof is merely a verification.

Our result also leads to the following result, that appears to be new: if $\mathcal{L}$ is closed under union, intersection and quotient, then its unambiguous polynomial

* Work supported by the project ANR 2010 BLAN 020202 FREC. 
closure and its left [right] deterministic polynomial closure are closed under the same operations.

Let us mention also that our algorithm can be readily extended to the setting of infinite words by using syntactic ordered $\omega$-semigroups [8].

\section{Background and notation}

\subsection{Syntactic order}

The syntactic congruence of a language $L$ of $A^{*}$ is the congruence on $A^{*}$ defined by $u \sim_{L} v$ if and only if, for every $x, y \in A^{*}$,

$$
x u y \in L \Longleftrightarrow x v y \in L
$$

The monoid $M=A^{*} / \sim_{L}$ is the syntactic monoid of $L$ and the natural morphism $\eta: A^{*} \rightarrow M$ is called the syntactic morphism of $L$. It is a well-known fact that a language is regular if and only if its syntactic monoid is finite.

The syntactic preorder 11 of a language $L$ is the relation $\leqslant_{L}$ over $A^{*}$ defined by $u \leqslant \leqslant_{L} v$ if and only if, for every $x, y \in A^{*}, x u y \in L$ implies $x v y \in L$. The associated equivalence relation is the syntactic congruence $\sim_{L}$. Further, $\leqslant_{L}$ induces a partial order on the syntactic monoid $M$ of $L$. This partial order $\leqslant$ is compatible with the product and can also be defined directly on $M$ as follows: given $s, t \in M$, one has $s \leqslant t$ if and only if, for all $x, y \in M, x s y \in \eta(L)$ implies $x t y \in \eta(L)$. The ordered monoid $(M, \leqslant)$ is called the syntactic ordered monoid of $L$.

Let us remind an elementary but useful fact: if $v \in L$ and $\eta(u) \leqslant \eta(v)$, then $u \in L$. This follows immediately form the definition of the syntactic order by taking $x=y=1$.

\subsection{Quotients}

Recall that if $L$ is a language of $A^{*}$ and $x$ is a word, the left quotient of $L$ by $x$ is the language $x^{-1} L=\left\{z \in A^{*} \mid x z \in L\right\}$. The right quotient $L y^{-1}$ is defined in a symmetrical way. Right and left quotients commute, and thus $x^{-1} L y^{-1}$ denotes either $x^{-1}\left(L y^{-1}\right)$ or $\left(x^{-1} L\right) y^{-1}$. For each word $v$, let us set

$$
\begin{aligned}
{[L]_{\uparrow v} } & =\left\{u \in A^{*} \mid \eta(v) \leqslant \eta(u)\right\} \\
{[L]_{=v} } & =\left\{u \in A^{*} \mid \eta(u)=\eta(v)\right\}
\end{aligned}
$$

\footnotetext{
${ }^{1}$ In earlier papers 61013, I used the opposite preorder, but it seems preferable to go back to Schützenberger's original definition.
} 
Proposition 2.1. The following formulas hold:

$$
\begin{aligned}
{[L]_{\uparrow v} } & =\bigcap_{\left\{(x, y) \in A^{*} \times A^{*} \mid v \in x^{-1} L y^{-1}\right\}} x^{-1} L y^{-1} \\
{[L]_{=v} } & =[L]_{\uparrow v}-\bigcup_{\eta(v)<\eta(u)}[L]_{\uparrow u} \\
{[L]_{\uparrow v} } & =\bigcup_{\eta(v) \leqslant \eta(u)}[L]_{=u}
\end{aligned}
$$

Proof. A word $u$ belongs to the right hand side of (11) if and only if the condition $v \in x^{-1} L y^{-1}$ implies $u \in x^{-1} L y^{-1}$, which is equivalent to stating that $v \leqslant_{L} u$, or $\eta(v) \leqslant \eta(u)$, or yet $u \in[L]_{\uparrow v}$. This proves (11). Formulas (2) and (3) are obvious.

Let us make precise a few critical points. First, $v$ always belongs to $[L]_{\uparrow v}$. This is the case even if $v$ cannot be completed into a word of $L$, that is, if $v$ does not belong to any quotient $x^{-1} L y^{-1}$. In this case, the intersection on the right hand side of (10) is indexed by the empty set and is therefore equal to $A^{*}$.

Secondly, the intersection occurring on the right hand side of (1) and the union occurring on the right hand side of (2) are potentially infinite, but they are finite if $L$ is a regular language, since a regular language has only finitely many quotients.

\section{Infiltration product and infiltration maps}

The definition below is a special case of a more general definition given in [7. A word $c_{1} \cdots c_{r}$ belongs to the infiltration product of two words $a_{1} \cdots a_{p}$ and $v=b_{1} \cdots b_{q}$, if there are two order preserving maps $\alpha:\{1, \ldots, p\} \rightarrow\{1, \ldots, r\}$ and $\beta:\{1, \ldots, q\} \rightarrow\{1, \ldots, r\}$ such that

(1) for each $i \in\{1, \ldots, p\}, a_{i}=c_{\alpha(i)}$,

(2) for each $i \in\{1, \ldots, q\}, b_{i}=c_{\beta(i)}$,

(3) the union of the ranges of $\alpha$ and $\beta$ is $\{1, \ldots, r\}$.

For instance, the set $\{a b, a a b, a b b, a a b b, a b a b\}$ is the infiltration product of $a b$ and $a b$ and the set $\{a b a, b a b, a b a b, a b b a, b a a b, b a b a\}$ is the infiltration product of $a b$ and $b a$.

A pair of maps $(\alpha, \beta)$ satisfying Conditions (1)-(3) is called a pair of infiltration maps. Note that these conditions imply that $p+q \leqslant r$.

In the example pictured in Figure 10 one has $p=4, q=2$ and $r=5$. The infiltration maps $\alpha$ and $\beta$ are given by $\alpha(1)=1, \alpha(2)=2, \alpha(3)=3, \alpha(4)=4$ and $\beta(1)=3, \beta(2)=5$. 


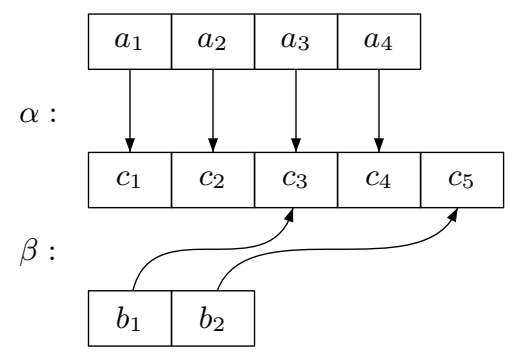

Fig. 1. A pair of infiltration maps.

In order to state our main theorem in a precise way, we need to handle the intervals of the form $\{\alpha(i)+1, \ldots, \alpha(i+1)-1\}$, but also the two extremal intervals $\{1, \ldots, \alpha(1)-1\}$ and $\{\alpha(p)+1, \ldots, r\}$. As a means to get a uniform notation, it is convenient to extend $\alpha$ and $\beta$ to mappings $\alpha:\{0, \ldots, p+1\} \rightarrow\{0, \ldots, r+1\}$ and $\beta:\{0, \ldots, q+1\} \rightarrow\{0, \ldots, r+1\}$ by setting $\alpha(0)=\beta(0)=0$ and $\alpha(p+1)=$ $\beta(q+1)=r+1$. The two extremal intervals are now of the standard form $\{\alpha(i)+$ $1, \ldots, \alpha(i+1)-1\}$, with $i=0$ and $i=p$, respectively. Further, we introduce the two maps $\bar{\alpha}:\{0, \ldots, r\} \rightarrow\{0, \ldots, p\}$ and $\bar{\beta}:\{0, \ldots, r\} \rightarrow\{0, \ldots, q\}$ defined by

$$
\bar{\alpha}(i)=\max \{k \mid \alpha(k) \leqslant i\} \quad \text { and } \quad \bar{\beta}(i)=\max \{k \mid \beta(k) \leqslant i\} .
$$

For instance, one gets for our example:

$$
\begin{array}{llllll}
\bar{\alpha}(0)=0 & \bar{\alpha}(1)=1 & \bar{\alpha}(2)=2 & \bar{\alpha}(3)=3 & \bar{\alpha}(4)=4 & \bar{\alpha}(5)=4 \\
\bar{\beta}(0)=0 & \bar{\beta}(1)=0 & \bar{\beta}(2)=0 & \bar{\beta}(3)=1 & \bar{\beta}(4)=1 & \bar{\beta}(5)=2
\end{array}
$$

These two functions are conveniently represented in Figure 2

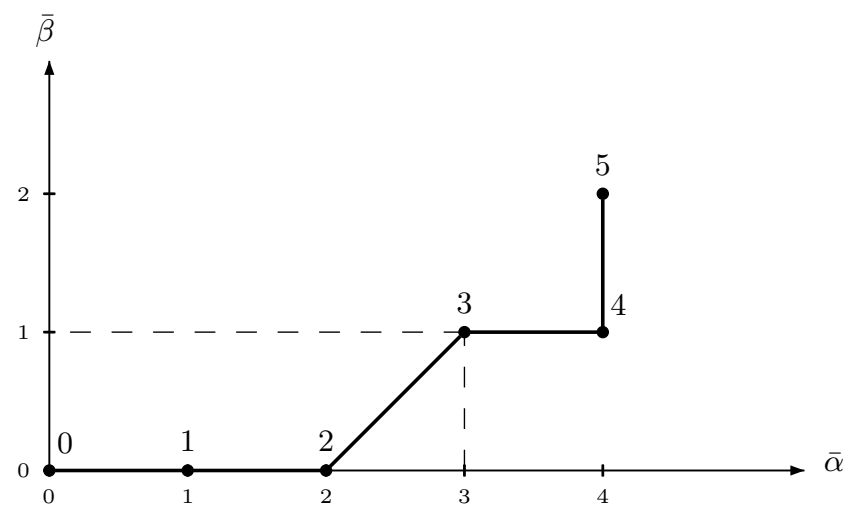

Fig. 2. Graphs of $\bar{\alpha}$ and $\bar{\beta}$ : for instance, $\bar{\alpha}(3)=3$ and $\bar{\beta}(3)=1$. 
The next lemmas summarize the connections between $\alpha$ and $\bar{\alpha}$. Of course, similar properties hold for $\beta$ and $\bar{\beta}$.

Lemma 3.1. The following properties hold:

(1) $\bar{\alpha}(\alpha(k))=k$, for $0 \leqslant k \leqslant p$.

(2) $\bar{\alpha}(s+1) \leqslant \bar{\alpha}(s)+1$, for $0 \leqslant s \leqslant r-1$.

(3) $k \leqslant \bar{\alpha}(s)$ if and only if $\alpha(k) \leqslant s$, for $0 \leqslant k \leqslant p$ and $0 \leqslant s \leqslant r$.

(4) $k \geqslant \bar{\alpha}(s)$ if and only if $\alpha(k+1) \geqslant s+1$, for $0 \leqslant k \leqslant p-1$ and $0 \leqslant s \leqslant r-1$.

Proof. These properties follow immediately from the definition of $\bar{\alpha}$.

Lemma 3.2. For $0 \leqslant s \leqslant r-1$, the conditions $\bar{\alpha}(s+1)=\bar{\alpha}(s)+1$ and $\alpha(\bar{\alpha}(s+1))=s+1$ are equivalent.

Proof. Put $k=\bar{\alpha}(s)$ and suppose that $\bar{\alpha}(s+1)=k+1$. Since $k+1 \leqslant \bar{\alpha}(s+1)$, Lemma 3.1 (3) shows that $\alpha(k+1) \leqslant s+1$. Further, since $k \geqslant \bar{\alpha}(s)$, Lemma 3.1 (4) shows that $\alpha(k+1) \geqslant s+1$. Therefore $\alpha(k+1)=s+1$ and finally $\alpha(\bar{\alpha}(s+1))=s+1$.

Conversely, suppose that $\alpha(\bar{\alpha}(s+1))=s+1$. Putting $\bar{\alpha}(s+1)=k+1$, one gets $\alpha(k+1)=s+1$ and Lemma 3.1 (4) shows that $k \geqslant \bar{\alpha}(s)$. By Lemma $3.1(2)$, one gets $\bar{\alpha}(s+1) \leqslant \bar{\alpha}(s)+1$ and hence $k \leqslant \bar{\alpha}(s)$. Thus $\bar{\alpha}(s)=k$ and $\bar{\alpha}(s+1)=\bar{\alpha}(s)+1$.

Let us denote by $P_{\alpha}(s)$ the property $\bar{\alpha}(s+1)=\bar{\alpha}(s)+1$.

Lemma 3.3. For $0 \leqslant s \leqslant r-1$, one of $P_{\alpha}(s)$ or $P_{\beta}(s)$ holds.

Proof. Since the union of the ranges of $\alpha$ and $\beta$ is $\{1, \ldots, r\}$, there is an integer $k \geqslant 0$ such that either $\alpha(k+1)=s+1$ or $\beta(k+1)=s+1$. In the first case, one gets $\bar{\alpha}(s+1)=\bar{\alpha}(\alpha(k+1))=k+1$ and Lemma $3.1(3)$ shows that $\bar{\alpha}(s) \leqslant k$. Since $\bar{\alpha}(s+1) \leqslant \bar{\alpha}(s)+1$ by Lemma 3.1 (2), one also has $k \leqslant \bar{\alpha}(s)$ and finally $\bar{\alpha}(s)=k$, which proves $P_{\alpha}(s)$. In the latter case, one gets $P_{\beta}(s)$ by a similar argument.

\section{Main result}

Let $a_{1}, \ldots, a_{p}, b_{1}, \ldots, b_{q}$ be letters of $A$ and let $K_{0}, \ldots, K_{p}, L_{0}, \ldots, L_{q}$ be languages of $A^{*}$. Let $K=K_{0} a_{1} K_{1} \cdots a_{p} K_{p}$ and $L=L_{0} b_{1} L_{1} \cdots b_{q} L_{q}$.

A word of $K \cap L$ can be factorized as $u_{0} a_{1} u_{1} \cdots a_{p} u_{p}$, with $u_{0} \in K_{0}, \ldots, u_{p} \in$ $K_{p}$ and as $v_{0} b_{1} v_{1} \cdots b_{q} v_{q}$, with $v_{0} \in L_{0}, \ldots, v_{q} \in L_{q}$. These two factorizations can be refined into a single factorization of the form $z_{0} c_{1} z_{1} \cdots c_{r} z_{r}$, where $c_{1} \cdots c_{r}$ belongs to the infiltration product of $a_{1} \cdots a_{p}$ and $b_{1} \cdots b_{q}$.

For instance, for $p=4$ and $q=2$, one could have $r=5$, with the relations $c_{1}=a_{1}, c_{2}=a_{2}, c_{3}=a_{3}=b_{1}, c_{4}=a_{4}$ and $c_{5}=b_{2}$, leading to the factorization $z_{0} c_{1} z_{1} c_{2} z_{2} c_{3} z_{3} c_{4} z_{4} c_{5} z_{5}$, as pictured in Figure 3 . 


\begin{tabular}{|l|l|l|l|l|l|l|l|l|}
\hline$u_{0}$ & $a_{1}$ & $u_{1}$ & $a_{2}$ & $u_{2}$ & $a_{3}$ & $u_{3}$ & $a_{4}$ & $u_{4}$ \\
\hline
\end{tabular}

\begin{tabular}{|l|l|l|l|l|l|l|l|l|l|l|}
\hline$z_{0}$ & $c_{1}$ & $z_{1}$ & $c_{2}$ & $z_{2}$ & $c_{3}$ & $z_{3}$ & $c_{4}$ & $z_{4}$ & $c_{5}$ & $z_{5}$ \\
\hline
\end{tabular}

\begin{tabular}{|l|l|l|l|l|}
\hline$v_{0}$ & $b_{1}$ & $v_{1}$ & $b_{2}$ & $v_{2}$ \\
\hline
\end{tabular}

Fig. 3. A word of $K \cap L$ and its factorizations.

The associated pair of infiltration maps $(\alpha, \beta)$ is given by

$$
\begin{array}{llll}
\alpha(1)=1 & \alpha(2)=2 & \alpha(3)=3 & \alpha(4)=4 \\
\beta(1)=3 & \beta(2)=5 & &
\end{array}
$$

Two series of constraints will be imposed on the words $z_{i}$ :

$$
\begin{aligned}
& z_{0} \in K_{0}, z_{1} \in K_{1}, z_{2} \in K_{2}, z_{3} \in K_{3} \text { and } z_{4} c_{5} z_{5} \in K_{4}, \\
& z_{0} c_{1} z_{1} c_{2} z_{2} \in L_{0}, z_{3} c_{4} z_{4} \in L_{1} \text { and } z_{5} \in L_{2} .
\end{aligned}
$$

We are now ready to state our main result. Let us denote by $I(p, q)$ the set of pairs of infiltration maps $(\alpha, \beta)$ with domain $\{1, \ldots, p\}$ and $\{1, \ldots, q\}$, respectively. Since $r \leqslant p+q$, the set $I(p, q)$ is finite.

Theorem 4.1. Let $K=K_{0} a_{1} K_{1} \cdots a_{p} K_{p}$ and $L=L_{0} b_{1} L_{1} \cdots b_{q} L_{q}$ be two products of languages. Then their intersection is given by the formulas

$$
K \cap L=\bigcup_{(\alpha, \beta) \in I(p, q)} U(\alpha, \beta)
$$

where

$$
U(\alpha, \beta)=\bigcup_{\left(z_{0}, \ldots, z_{r}\right) \in C(\alpha, \beta)} U_{0} c_{1} U_{1} \cdots c_{r} U_{r}
$$

and, for $0 \leqslant i \leqslant r$,

$$
U_{i}=\left[K_{\bar{\alpha}(i)}\right]_{\uparrow z_{i}} \cap\left[L_{\bar{\beta}(i)}\right]_{\uparrow z_{i}}
$$

and $C(\alpha, \beta)$ is the set of $(r+1)$-tuples $\left(z_{0}, \ldots, z_{r}\right)$ of words such that

$\left(\mathrm{C}_{1}\right)$ for $0 \leqslant k \leqslant p, z_{\alpha(k)} c_{\alpha(k)+1} z_{\alpha(k)+1} \cdots c_{\alpha(k+1)-1} z_{\alpha(k+1)-1} \in K_{k}$,

$\left(\mathrm{C}_{2}\right)$ for $0 \leqslant k \leqslant q, z_{\beta(k)} c_{\beta(k)+1} z_{\beta(k)+1} \cdots c_{\beta(k+1)-1} z_{\beta(k+1)-1} \in L_{k}$.

For instance, if $(\alpha, \beta)$ is the pair of infiltration maps of our example, one would have

$$
\begin{gathered}
U(\alpha, \beta)=\bigcup_{\left(z_{0}, \ldots, z_{5}\right) \in C(\alpha, \beta)}\left(\left[K_{0}\right]_{\uparrow z_{0}} \cap\left[L_{0}\right]_{\uparrow z_{0}}\right) a_{1}\left(\left[K_{1}\right]_{\uparrow z_{1}} \cap\left[L_{0}\right]_{\uparrow z_{1}}\right) a_{2} \\
\left(\left[K_{2}\right]_{\uparrow z_{2}} \cap\left[L_{0}\right]_{\uparrow z_{2}}\right) b_{1}\left(\left[K_{3}\right]_{\uparrow z_{3}} \cap\left[L_{1}\right]_{\uparrow z_{3}}\right) a_{4}\left(\left[K_{4}\right]_{\uparrow z_{4}} \cap\left[L_{1}\right]_{\uparrow z_{4}}\right) b_{2}\left(\left[K_{4}\right]_{\uparrow z_{5}} \cap\left[L_{2}\right]_{\uparrow z_{5}}\right)
\end{gathered}
$$


and the conditions $\left(\mathrm{C}_{1}\right)$ and $\left(\mathrm{C}_{2}\right)$ would be

$\left(\mathrm{C}_{1}\right) z_{0} \in K_{0}, z_{1} \in K_{1}, z_{2} \in K_{2}, z_{3} \in K_{3}, z_{4} c_{5} z_{5} \in K_{4}$,

$\left(\mathrm{C}_{2}\right) z_{0} c_{1} z_{1} c_{2} z_{2} \in L_{0}, z_{3} c_{4} z_{4} \in L_{1}$ and $z_{5} \in L_{2}$.

Before proving the theorem, it is important to note that if the languages $K_{0}$, $\ldots, K_{p}, L_{0}, \ldots, L_{q}$ are regular, the union indexed by $C(\alpha, \beta)$ is actually a finite union. Indeed, Proposition 2.1 shows that, if $R$ is a regular language, there are only finitely many languages of the form $[R]_{z}$.

Proof. Let $U$ be the right hand side of (4). We first prove that $K \cap L$ is a subset of $U$. Let $z$ be a word of $K \cap L$. Then $z$ can be factorized as $u_{0} a_{1} u_{1} \cdots a_{p} u_{p}$, with $u_{0} \in K_{0}, \ldots, u_{p} \in K_{p}$ and as $v_{0} b_{1} v_{1} \cdots b_{q} v_{q}$, with $v_{0} \in L_{0}, \ldots, v_{q} \in L_{q}$. The common refinement of these two factorizations leads to a factorization of the form $z_{0} c_{1} z_{1} \cdots c_{r} z_{r}$, where each letter $c_{k}$ is either equal to some $a_{i}$ or to some $b_{j}$ or both. This naturally defines a pair of infiltration maps $\alpha:\{1, \ldots, p\} \rightarrow\{1, \ldots, r\}$ and $\beta:\{1, \ldots, q\} \rightarrow\{1, \ldots, r\}$. Conditions $\left(\mathrm{C}_{1}\right)$ and $\left(\mathrm{C}_{2}\right)$ just say that the factorization $z_{0} c_{1} z_{1} \cdots c_{r} z_{r}$ is a refinement of the two other ones. Now, since, for $0 \leqslant i \leqslant r$, the word $z_{i}$ belongs to $\left[K_{\bar{\alpha}(i)}\right]_{\uparrow z_{i}} \cap\left[L_{\bar{\beta}(i)}\right]_{\uparrow z_{i}}$, the word $z$ belongs to $U$. Thus $K \cap L \subseteq U$.

We now prove the opposite inclusion. Let $r \leqslant p+q$ be an integer, let $\alpha$ : $\{1, \ldots, p\} \rightarrow\{1, \ldots, r\}$ and $\beta:\{1, \ldots, q\} \rightarrow\{1, \ldots, r\}$ be two infiltration maps and let $\left(z_{0}, \ldots, z_{r}\right) \in C(\alpha, \beta)$ and $c_{1}, \ldots, c_{r}$ satisfying $\left(\mathrm{C}_{1}\right)$ and $\left(\mathrm{C}_{2}\right)$. It suffices to prove that $U_{0} c_{1} U_{1} \cdots c_{r} U_{r}$ is a subset of $K \cap L$. We need a stronger version of $\left(\mathrm{C}_{1}\right)$ and $\left(\mathrm{C}_{2}\right)$.

Lemma 4.2. The following relations hold:

$\left(\mathrm{C}_{3}\right)$ for $0 \leqslant k \leqslant p, U_{\alpha(k)} c_{\alpha(k)+1} U_{\alpha(k)+1} \cdots c_{\alpha(k+1)-1} U_{\alpha(k+1)-1} \subseteq K_{k}$,

$\left(\mathrm{C}_{4}\right)$ for $0 \leqslant k \leqslant q, U_{\beta(k)} c_{\beta(k)+1} U_{\beta(k)+1} \cdots c_{\beta(k+1)-1} U_{\beta(k+1)-1} \subseteq L_{k}$.

Coming back once again to our main example, these conditions would be

$\left(\mathrm{C}_{3}\right) U_{0} \subseteq K_{0}, U_{1} \subseteq K_{1}, U_{2} \subseteq K_{2}, U_{3} \subseteq K_{3}, U_{4} c_{4} U_{5} \subseteq K_{4}$,

$\left(\mathrm{C}_{4}\right) U_{0} c_{1} U_{2} c_{2} U_{2} \subseteq L_{0}, U_{3} c_{4} U_{4} \subseteq L_{1}, U_{5} \subseteq L_{5}$.

Proof. Let $\eta_{k}$ be the syntactic morphism of $K_{k}$. To simplify notation, let us set $i=\alpha(k)+1$ and $j=\alpha(k+1)-1$. Since $\alpha(k)=i-1<i<\cdots<j<\alpha(k+1)$, one gets $\bar{\alpha}(i-1)=\bar{\alpha}(i)=\ldots=\bar{\alpha}(j)=k$. Let $u_{i-1} \in U_{i-1}, u_{i} \in U_{i}, \ldots$, $u_{j} \in U_{j}$. Then $u_{i-1} \in\left[U_{k}\right]_{\uparrow z_{i-1}}, u_{i} \in\left[U_{k}\right]_{\uparrow z_{i}}, \ldots, u_{j} \in\left[U_{k}\right]_{\uparrow z_{j}}$ and by definition, $\eta_{k}\left(z_{i-1}\right) \leqslant \eta_{k}\left(u_{i-1}\right), \eta_{k}\left(z_{i}\right) \leqslant \eta_{k}\left(u_{i}\right), \ldots, \eta_{k}\left(z_{j}\right) \leqslant \eta_{k}\left(u_{j}\right)$. Therefore we get

$$
\begin{aligned}
& \eta_{k}\left(z_{i-1} c_{i} z_{i} \cdots c_{j} z_{j}\right)=\eta_{k}\left(z_{i-1}\right) \eta_{k}\left(c_{i}\right) \eta_{k}\left(z_{i}\right) \cdots \eta_{k}\left(c_{j}\right) \eta_{k}\left(z_{j}\right) \\
& \quad \leqslant \eta_{k}\left(u_{i-1}\right) \eta_{k}\left(c_{i}\right) \eta_{k}\left(u_{i}\right) \cdots \eta_{k}\left(c_{j}\right) \eta_{k}\left(u_{j}\right)=\eta_{k}\left(u_{i-1} c_{i} u_{i} \cdots c_{j} u_{j}\right)
\end{aligned}
$$

Now, since $z_{i-1} c_{i} z_{i} \cdots c_{j} z_{j} \in K_{k}$ by $\left(\mathrm{C}_{1}\right)$, we also get $u_{i-1} c_{i} u_{i} \cdots c_{j} u_{j} \in K_{k}$, which proves $\left(\mathrm{C}_{3}\right)$. The proof of $\left(\mathrm{C}_{4}\right)$ is similar.

Now, since $\bar{\alpha}$ and $\bar{\beta}$ are surjective, Lemma 4.2 shows that $U_{0} c_{1} U_{1} \cdots c_{r} U_{r}$ is a subset of $K \cap L$, which concludes the proof of the theorem. 
Example 4.3. Let $K=b^{*} a A^{*} b a^{*}$ and $L=a^{*} b A^{*} a b^{*}$. The algorithm described in Theorem 4.1 gives for $K \cap L$ the expression $a a^{*} b A^{*} b a^{*} a \cup b b^{*} a A^{*} b a^{*} a \cup$ $a a^{*} b A^{*} a b^{*} b \cup b b^{*} a A^{*} a b^{*} b \cup a a^{*} b a^{*} a \cup b b^{*} a b^{*} b$.

Corollary 4.4. Let $\mathcal{L}$ be a lattice of regular languages closed under quotient. Then its polynomial closure is also a lattice closed under quotient.

\section{Some variants of the product}

We consider in this section two variants of the product introduced by Schützenberger in [15]: unambiguous and deterministic products. These products were also stud-

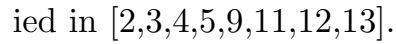

\subsection{Unambiguous product}

The marked product $L=L_{0} a_{1} L_{1} \cdots a_{n} L_{n}$ of $n$ nonempty languages $L_{0}, L_{1}, \ldots$, $L_{n}$ of $A^{*}$ is unambiguous if every word $u$ of $L$ admits a unique factorization of the form $u_{0} a_{1} u_{1} \cdots a_{n} u_{n}$ with $u_{0} \in L_{0}, u_{1} \in L_{1}, \ldots, u_{n} \in L_{n}$. We require the languages $L_{i}$ to be nonempty to make sure that subfactorizations remain unambiguous:

Proposition 5.1. Let $L_{0} a_{1} L_{1} \cdots a_{n} L_{n}$ be an unambiguous product and let $i_{1}$, $\ldots, i_{k}$ be a sequence of integers satisfying $0<i_{1}<\ldots<i_{k}<n$. Finally, let $R_{0}=L_{0} a_{1} L_{1} \cdots a_{i_{1}-1} L_{i_{1}-1}, R_{1}=L_{i_{1}} a_{i_{1}+1} L_{1} \cdots a_{i_{2}-1} L_{i_{2}-1}, \ldots, R_{k}=$ $L_{i_{k}} a_{i_{k}+1} L_{i_{k}+1} \cdots a_{n} L_{n}$. Then the product $R_{0} a_{i_{1}} R_{1} \cdots a_{i_{k}} R_{k}$ is unambiguous.

Proof. Trivial.

The unambiguous polynomial closure of a class of languages $\mathcal{L}$ of $A^{*}$ is the set of languages that are finite unions of unambiguous products of the form $L_{0} a_{1} L_{1} \cdots a_{n} L_{n}$, where the $a_{i}$ 's are letters and the $L_{i}$ 's are elements of $\mathcal{L}$. The term closure actually requires a short justification.

Proposition 5.2. Any unambiguous product of unambiguous products is unambiguous.

Proof. Let

$$
\begin{aligned}
L_{0} & =L_{0,0} a_{1,0} L_{1,0} \cdots a_{k_{0}, 0} L_{k_{0}, 0} \\
L_{1} & =L_{0,1} a_{1,1} L_{1,1} \cdots a_{k_{1}, 1} L_{k_{1}, 1} \\
& \vdots \\
L_{n} & =L_{0, n} a_{1, n} L_{1, n} \cdots a_{k_{n}, n} L_{k_{n}, n}
\end{aligned}
$$

be unambiguous products and let $L=L_{0} b_{1} L_{1} \cdots b_{n} L_{n}$ be an unambiguous product. We claim that the product

$$
L_{0,0} a_{1,0} L_{1,0} \cdots a_{k_{0}, 0} L_{k_{0}, 0} b_{1} L_{0,1} a_{1,1} L_{1,1} \cdots b_{n} L_{0, n} a_{1, n} L_{1, n} \cdots a_{k_{n}, n} L_{k_{n}, n}
$$


is unambiguous. Let $u$ be a word of $L$ with two factorizations

$$
x_{0,0} a_{1,0} x_{1,0} \cdots a_{k_{0}, 0} x_{k_{0}, 0} b_{1} x_{0,1} a_{1,1} x_{1,1} \cdots b_{n} x_{0, n} a_{1, n} x_{1, n} \cdots a_{k_{n}, n} x_{k_{n}, n}
$$

and

$$
y_{0,0} a_{1,0} y_{1,0} \cdots a_{k_{0}, 0} y_{k_{0}, 0} b_{1} y_{0,1} a_{1,1} x_{1,1} \cdots b_{n} y_{0, n} a_{1, n} y_{1, n} \cdots a_{k_{n}, n} y_{k_{n}, n}
$$

with $x_{0,0}, y_{0,0} \in L_{0,0}, \ldots, x_{k_{n}, n}, y_{k_{n}, n} \in L_{k_{n}, n}$. Setting

$$
\begin{array}{rlrl}
x_{0} & =x_{0,0} a_{1,0} x_{1,0} \cdots a_{k_{0}, 0} x_{k_{0}, 0} & y_{0} & =y_{0,0} a_{1,0} y_{1,0} \cdots a_{k, 0} y_{k_{0}, 0} \\
x_{1} & =x_{0,1} a_{1,1} x_{1,1} \cdots a_{k_{1}, 1} x_{k_{1}, 1} & y_{1} & =y_{0,1} a_{1,1} y_{1,1} \cdots a_{k_{1}, 1} y_{k_{1}, 1} \\
& \vdots & \vdots \\
x_{n} & =x_{0, n} a_{1, n} x_{1, n} \cdots a_{k_{n}, n} x_{k_{n}, n} & y_{n}=y_{0, n} a_{1, n} y_{1, n} \cdots a_{k_{n}, n} y_{k_{n}, n}
\end{array}
$$

we get two factorizations of $u$ : $x_{0} b_{1} x_{1} \cdots b_{n} x_{n}$ and $y_{0} b_{1} y_{1} \cdots b_{n} y_{n}$. Since the product $L_{0} b_{1} L_{1} \cdots a_{n} L_{n}$ is unambiguous, we have $x_{0}=y_{0}, \ldots, x_{n}=y_{n}$. Each of these words has now two factorizations given by (8) and since the products of (77) are unambiguous, these factorizations are equal. This proves the claim and the proposition.

We now consider the intersection of two unambiguous products.

Theorem 5.3. If the products $K=K_{0} a_{1} K_{1} \cdots a_{p} K_{p}$ and $L=L_{0} b_{1} L_{1} \cdots b_{q} L_{q}$ are unambiguous, the products occurring in Formula (4) are all unambiguous.

Proof. Let $(\alpha, \beta)$ be a pair of infiltration maps, and let $U_{i}=\left[K_{\bar{\alpha}(i)}\right] \uparrow z_{i} \cap\left[L_{\bar{\beta}(i)}\right]_{\uparrow z_{i}}$, for $0 \leqslant i \leqslant r$. We claim that the product $U=U_{0} c_{1} U_{1} \cdots c_{r} U_{r}$ is unambiguous. Let

$$
u=u_{0} c_{1} u_{1} \cdots c_{r} u_{r}=u_{0}^{\prime} c_{1} u_{1}^{\prime} \cdots c_{r} u_{r}^{\prime}
$$

be two factorizations of a word $u$ of $U$ such that, for $0 \leqslant i \leqslant r, u_{i}, u_{i}^{\prime} \in U_{i}$. We prove by induction on $s$ that $u_{s}=u_{s}^{\prime}$.

Case $s=0$. By the properties of $\alpha$ and $\beta$, we may assume without loss of generality that $\alpha(1)=1$, which implies that $c_{1}=a_{1}$. It follows from $\left(\mathrm{C}_{3}\right)$ that $U_{0} \subseteq K_{0}$. Now the product $K_{0} a_{1}\left(K_{1} a_{2} K_{2} \cdots a_{p} K_{p}\right)$ is unambiguous by Proposition 5.1. and by $\left(\mathrm{C}_{3}\right), U_{1} c_{2} U_{2} \cdots c_{r} U_{r}$ is contained in $K_{1} a_{1} K_{2} \cdots a_{p} K_{p}$. Therefore, $u$ admits the two factorizations $u_{0} a_{1}\left(u_{1} c_{2} u_{2} \cdots c_{r} u_{r}\right)$ and $u_{0}^{\prime} a_{1}\left(u_{1}^{\prime} c_{2} u_{2}^{\prime} \cdots c_{r} u_{r}^{\prime}\right)$ in this product. Thus $u_{0}=u_{0}^{\prime}$.

Induction step. Let $s>0$ and suppose by induction that $u_{i}=u_{i}^{\prime}$ for $0 \leqslant i \leqslant s-1$. If $s=r$, then necessarily $u_{s}=u_{s}^{\prime}$. If $s<r$, we may assume without loss of generality that $s$ is in the range of $\alpha$. Thus $\alpha(k)=s$ for some $k$ and $c_{s}=a_{k}$. We now consider two cases separately.

If $\alpha(k+1)=s+1$ (and $\left.c_{s+1}=a_{k+1}\right)$, it follows from $\left(\mathrm{C}_{3}\right)$ that $u$ has two factorizations

$$
\begin{aligned}
& \left(u_{0} c_{1} u_{1} \cdots c_{s-1} u_{s-1}\right) a_{k} u_{s} a_{k+1}\left(u_{s+1} c_{s+1} u_{s+2} \cdots c_{r} u_{r}\right) \text { and } \\
& \left(u_{0} c_{1} u_{1} \cdots c_{s-1} u_{s-1}\right) a_{k} u_{s}^{\prime} a_{k+1}\left(u_{s+1}^{\prime} c_{s+1} u_{s+2}^{\prime} \cdots c_{r} u_{r}^{\prime}\right)
\end{aligned}
$$


over the product $\left(K_{0} a_{1} K_{1} \cdots a_{s-1} K_{s-1}\right) a_{k} K_{s} a_{k+1}\left(K_{s+1} a_{k+2} K_{s+2} \cdots a_{p} K_{p}\right)$. Since this product is unambiguous by Proposition 5.1, we get $u_{s}=u_{s}^{\prime}$.

If $\alpha(k+1) \neq s+1$, then $s+1=\beta(t+1)$ for some $t$ and $c_{s+1}=b_{t+1}$. Setting $i=\beta(t)$, we get $c_{i}=b_{t}$ and it follows from $\left(\mathrm{C}_{4}\right)$ that $u$ has two factorizations

$$
\begin{array}{r}
\left(u_{0} c_{1} u_{1} \cdots c_{i-1} u_{i-1}\right) b_{t}\left(u_{i} c_{i+1} u_{i+1} \cdots c_{s} u_{s}\right) b_{t+1}\left(u_{s+1} c_{s+2} u_{s+2} \cdots c_{r} u_{r}\right) \text { and } \\
\left(u_{0} c_{1} u_{1} \cdots c_{i-1} u_{i-1}\right) b_{t}\left(u_{i}^{\prime} c_{i+1} u_{i+1}^{\prime} \cdots c_{s} u_{s}^{\prime}\right) b_{t+1}\left(u_{s+1}^{\prime} c_{s+2} u_{s+2}^{\prime} \cdots c_{r} u_{r}^{\prime}\right)
\end{array}
$$

over the product $\left(L_{0} b_{1} L_{1} \cdots b_{t-1} L_{t-1}\right) b_{t} L_{t} b_{t+1}\left(L_{t+1} b_{t+1} L_{t+2} \cdots b_{p} L_{p}\right)$. This product is unambiguous by Proposition 5.1, and thus

$$
u_{i} c_{i+1} u_{i+1} \cdots c_{s} u_{s}=u_{i}^{\prime} c_{i+1} u_{i+1}^{\prime} \cdots c_{s} u_{s}^{\prime}
$$

Now the induction hypothesis gives $u_{i}=u_{i}^{\prime}, \ldots, u_{s-1}=u_{s-1}^{\prime}$ and one finally gets $u_{s}=u_{s}^{\prime}$.

We state separately another interesting property.

Theorem 5.4. Let $K=K_{0} a_{1} K_{1} \cdots a_{p} K_{p}$ and $L=L_{0} b_{1} L_{1} \cdots b_{q} L_{q}$ be two unambiguous products and let $(\alpha, \beta)$ and $\left(\alpha^{\prime}, \beta^{\prime}\right)$ be two pairs of infiltration maps of $I(p, q)$. If the sets $U(\alpha, \beta)$ and $U\left(\alpha^{\prime}, \beta^{\prime}\right)$ meet, then $\alpha=\alpha^{\prime}$ and $\beta=\beta^{\prime}$.

Proof. Suppose that a word $u$ belongs to $U(\alpha, \beta)$ and to $U\left(\alpha^{\prime}, \beta^{\prime}\right)$. Then $u$ has two decompositions of the form

$$
u=u_{0} c_{1} u_{1} \cdots c_{r} u_{r}=u_{0}^{\prime} c_{1}^{\prime} u_{1}^{\prime} \cdots c_{r^{\prime}}^{\prime} u_{r^{\prime}}^{\prime}
$$

Condition $\left(\mathrm{C}_{1}\right)\left[\left(\mathrm{C}_{2}\right)\right]$ and the unambiguity of the product $K_{0} a_{1} K_{1} \cdots a_{p} K_{p}$ $\left[L_{0} b_{1} L_{1} \cdots b_{q} L_{q}\right]$ show that, for $0 \leqslant i \leqslant p$ and for $0 \leqslant j \leqslant q$,

$$
\begin{aligned}
& u_{\alpha(i)} c_{\alpha(i)+1} u_{\alpha(i)+1} \cdots c_{\alpha(i+1)-1} u_{\alpha(i+1)-1}= \\
& u_{\alpha^{\prime}(i)}^{\prime} c_{\alpha^{\prime}(i)+1}^{\prime} u_{\alpha^{\prime}(i)+1}^{\prime} \cdots c_{\alpha^{\prime}(i+1)-1} u_{\alpha^{\prime}(i+1)-1} \in K_{i} \\
& u_{\beta(j)} c_{\beta(j)+1} u_{\beta(j)+1} \cdots c_{\beta(j+1)-1} u_{\beta(j+1)-1}= \\
& u_{\beta^{\prime}(j)}^{\prime} c_{\beta^{\prime}(j)+1}^{\prime} u_{\beta^{\prime}(j)+1}^{\prime} \cdots c_{\beta^{\prime}(j+1)-1} u_{\beta^{\prime}(j+1)-1} \in L_{j}
\end{aligned}
$$

We prove by induction on $s$ that, for $1 \leqslant s \leqslant \min \left(r, r^{\prime}\right)$, the following properties hold:

$\mathrm{E}_{1}(s): u_{s-1}=u_{s-1}^{\prime}$ and $c_{s}=c_{s}^{\prime}$,

$\mathrm{E}_{2}(s): \bar{\alpha}(s)=\bar{\alpha}^{\prime}(s)$ and $\bar{\beta}(s)=\bar{\beta}^{\prime}(s)$,

$\mathrm{E}_{3}(s)$ : for $i \leqslant \bar{\alpha}(s), \alpha(i)=\alpha^{\prime}(i)$ and for $j \leqslant \bar{\beta}(s), \beta(j)=\beta^{\prime}(j)$.

Case $s=1$. We know that either $\alpha(1)=1$ or $\beta(1)=1$ and that either $\alpha^{\prime}(1)=1$ or $\beta^{\prime}(1)=1$. Suppose that $\alpha(1)=1$. We claim that $\alpha^{\prime}(1)=1$. Otherwise, one has $\beta^{\prime}(1)=1$. Now, Formula (10) applied to $i=0$ gives

$$
u_{0}=u_{0}^{\prime} c_{1}^{\prime} u_{1}^{\prime} \cdots c_{\alpha^{\prime}(1)-1} u_{\alpha^{\prime}(1)-1}^{\prime}
$$


and Formula (11) applied to $j=0$ gives

$$
u_{0} c_{1} u_{1} \cdots c_{\beta(1)-1} u_{\beta(1)-1}=u_{0}^{\prime} .
$$

Therefore $u_{0}=u_{0}^{\prime}$ and $\alpha^{\prime}(1)=1$, which proves the claim. It follows also that $a_{1}=c_{\alpha(1)}=c_{\alpha^{\prime}(1)}$ and thus $c_{1}=c_{1}^{\prime}$. We also have in this case $\bar{\alpha}(1)=\bar{\alpha}^{\prime}(1)=1$. A similar argument shows that if $\alpha^{\prime}(1)=1$, then $\alpha(1)=1$. Therefore, the conditions $\alpha(1)=1$ and $\alpha^{\prime}(1)=1$ are equivalent and it follows that $\bar{\alpha}(1)=\bar{\alpha}^{\prime}(1)$. A dual argument would prove that the conditions $\beta(1)=1$ and $\beta^{\prime}(1)=1$ are equivalent and that $\bar{\beta}(1)=\bar{\beta}^{\prime}(1)$.

Induction step. Let $s$ be such that $1 \leqslant s+1 \leqslant \min \left(r, r^{\prime}\right)$ and suppose by induction that the properties $\mathrm{E}_{1}(i), \mathrm{E}_{2}(i), \mathrm{E}_{3}(i)$ hold for $1 \leqslant i \leqslant s$.

Lemma 5.5. Suppose that $P_{\alpha}(s)$ holds and let $k=\bar{\alpha}(s)$. Then

$$
s \leqslant \alpha^{\prime}(k+1)-1
$$

and

$$
u_{s}=u_{s}^{\prime} c_{s+1}^{\prime} u_{s+1}^{\prime} \cdots c_{\alpha^{\prime}(k+1)-1} u_{\alpha^{\prime}(k+1)-1}^{\prime}
$$

Proof. Applying (10) with $i=k$, we get

$$
\begin{aligned}
& u_{\alpha(k)} c_{\alpha(k)+1} u_{\alpha(k)+1} \cdots c_{s} u_{s}= \\
& u_{\alpha^{\prime}(k)}^{\prime} c_{\alpha^{\prime}(k)+1}^{\prime} u_{\alpha^{\prime}(k)+1}^{\prime} \cdots c_{\alpha^{\prime}(k+1)-1} u_{\alpha^{\prime}(k+1)-1}^{\prime}
\end{aligned}
$$

Since $\bar{\alpha}(s)=\bar{\alpha}^{\prime}(s)$ by $\mathrm{E}_{2}(s)$, one has $\bar{\alpha}^{\prime}(s)=k$ and $\alpha^{\prime}(k+1) \geqslant s+1$ by Lemma 3.1, which gives (12). Further, since $k=\bar{\alpha}(s)$, it follows from $\mathrm{E}_{3}(s)$ that $\alpha(k)=\alpha^{\prime}(k)$. Now, for $i \leqslant s, \mathrm{E}_{1}(i)$ implies that $u_{i-1}=u_{i-1}^{\prime}$ and $c_{i}=c_{i}^{\prime}$. It follows that the word $u_{\alpha(k)} c_{\alpha(k)+1} u_{\alpha(k)+1} \cdots c_{s}$ is a prefix of both sides of (14). Therefore, this prefix can be deleted from both sides of (14), which gives (13).

We now establish $\mathrm{E}_{1}(s+1)$.

Lemma 5.6. One has $u_{s}=u_{s}^{\prime}$ and $c_{s+1}=c_{s+1}^{\prime}$. Further, $P_{\alpha}(s)$ and $P_{\alpha^{\prime}}(s)$ are equivalent and $P_{\beta}(s)$ and $P_{\beta^{\prime}}(s)$ are equivalent.

Proof. Let us prove that $u_{s}^{\prime}$ is a prefix of $u_{s}$. By Lemma 3.3 either $P_{\alpha}(s)$ or $P_{\beta}(s)$ holds. Suppose that $P_{\alpha}(s)$ holds. Then by Lemma 5.5, $u_{s}^{\prime}$ is a prefix of $u_{s}$. If $P_{\beta}(s)$ holds, we arrive to the same conclusion by using (11) in place of (10) in the proof of Lemma 5.5 .

Now, a symmetrical argument using the pair $\left(\bar{\alpha}^{\prime}, \bar{\beta}^{\prime}\right)$ would show that $u_{s}$ is a prefix of $u_{s}^{\prime}$. Therefore, $u_{s}=u_{s}^{\prime}$. Coming back to (13), we obtain $\alpha^{\prime}(k+1)=s+1$ and since by $\mathrm{E}_{2}(s), k=\bar{\alpha}(s)=\bar{\alpha}^{\prime}(s)$, one gets $\alpha^{\prime}\left(\bar{\alpha}^{\prime}(s)+1\right)=s+1$, which, by Lemma 3.2, is equivalent to $P_{\alpha^{\prime}}(s)$. Thus $P_{\alpha}(s)$ implies $P_{\alpha^{\prime}}(s)$ and a dual argument would prove the opposite implication.

We also have $c_{s+1}=c_{\alpha(k+1)}=a_{k+1}=c_{\alpha^{\prime}(k+1)}^{\prime}=c_{s+1}^{\prime}$ and thus $c_{s+1}=c_{s+1}^{\prime}$. Finally, a similar argument works for $\beta$. 
We now come to the proof of $\mathrm{E}_{2}(s+1)$ and $\mathrm{E}_{3}(s+1)$. Since $P_{\alpha}(s)$ and $P_{\alpha^{\prime}}(s)$ are equivalent, the next two lemma cover all cases.

Lemma 5.7. If neither $P_{\alpha}(s)$ nor $P_{\alpha^{\prime}}(s)$ hold, then $\bar{\alpha}(s+1)=\bar{\alpha}^{\prime}(s+1)$ and for $i \leqslant \bar{\alpha}(s+1), \alpha(i)=\alpha^{\prime}(i)$. Similarly, if neither $P_{\beta}(s)$ nor $P_{\beta^{\prime}}(s)$ hold, then $\bar{\beta}(s+1)=\bar{\beta}^{\prime}(s+1)$ and for $i \leqslant \bar{\beta}(s+1), \beta(i)=\beta^{\prime}(i)$.

Proof. We just prove the " $\alpha$ part" of the lemma. If neither $P_{\alpha}(s)$ nor $P_{\alpha^{\prime}}(s)$ hold, then $\bar{\alpha}(s+1)=\bar{\alpha}(s)$ and $\bar{\alpha}^{\prime}(s+1)=\bar{\alpha}^{\prime}(s)$. Since $\bar{\alpha}(s)=\bar{\alpha}^{\prime}(s)$ by $\mathrm{E}_{2}(s)$, one gets $\bar{\alpha}(s+1)=\bar{\alpha}^{\prime}(s+1)$. The second property is an immediate consequence of $\mathrm{E}_{3}(s)$.

Lemma 5.8. If both $P_{\alpha}(s)$ and $P_{\alpha^{\prime}}(s)$ hold, then $\bar{\alpha}(s+1)=\bar{\alpha}^{\prime}(s+1)$ and for $i \leqslant \bar{\alpha}(s+1), \alpha(i)=\alpha^{\prime}(i)$. Similarly, if both $P_{\beta}(s)$ and $P_{\beta^{\prime}}(s)$ hold, then $\bar{\beta}(s+1)=\bar{\beta}^{\prime}(s+1)$ and for $i \leqslant \bar{\beta}(s+1), \beta(i)=\beta^{\prime}(i)$.

Proof. Again, we just prove the " $\alpha$ part" of the lemma. If both $P_{\alpha}(s)$ and $P_{\alpha^{\prime}}(s)$ hold, then $\bar{\alpha}(s+1)=\bar{\alpha}(s)+1$ and $\bar{\alpha}^{\prime}(s+1)=\bar{\alpha}^{\prime}(s)+1$. Since $\bar{\alpha}(s)=\bar{\alpha}^{\prime}(s)$ by $\mathrm{E}_{2}(s)$, one gets $\bar{\alpha}(s+1)=\bar{\alpha}^{\prime}(s+1)$. Property $\mathrm{E}_{3}(s)$ shows that for $i \leqslant \bar{\alpha}(s)$, $\alpha(i)=\alpha^{\prime}(i)$. Since $\bar{\alpha}(s+1)=\bar{\alpha}(s)+1$, it just remains to prove that

$$
\alpha(\bar{\alpha}(s+1))=\alpha^{\prime}(\bar{\alpha}(s+1))
$$

But Lemma 3.2 shows that $\alpha(\bar{\alpha}(s+1))=s+1$ and $\alpha^{\prime}\left(\bar{\alpha}^{\prime}(s+1)\right)=s+1$, which proves (15) since $\bar{\alpha}(s+1)=\bar{\alpha}^{\prime}(s+1)$.

This concludes the induction step and the proof of Theorem 5.4 $\square$

Corollary 5.9. Let $\mathcal{L}$ be a lattice of regular languages closed under quotient. Then its unambiguous polynomial closure is also a lattice closed under quotient.

If $\mathcal{L}$ is a Boolean algebra, then one can be more precise.

Corollary 5.10. Let $\mathcal{L}$ be a Boolean algebra of regular languages closed under quotient. Then its unambiguous polynomial closure is also a Boolean algebra closed under quotient.

Let us conclude with an example which shows that, under the assumptions of Theorem 5.4, the sets $U(\alpha, \beta)$ cannot be further decomposed as a disjoint union of unambiguous products.

Let $K=K_{0} a K_{1}$ and $L=L_{0} a L_{1}$ with $K_{0}=L_{1}=1+b+c+c^{2}$ and $L_{0}=K_{1}=$ $a+a b+b a+a c+c a+a c^{2}+b a b+c a c+c a c^{2}$. Then

$$
\begin{gathered}
K \cap L=a a+a a b+a b a+a a c+a c a+a a c^{2}+a b a b+a c a c+a c a c^{2}+ \\
b a a+b a a b+b a b a+b a a c+b a a c^{2}+b a b a b+c a a+ \\
c a a b+c a a c+c a c a+c a a c^{2}+c a c a c+c a c a c^{2}
\end{gathered}
$$

One can write for instance $K \cap L$ as $(1+b+c) a a\left(1+b+c+c^{2}\right)+(1+b) a(1+$ b) $a(1+b)+(1+c) a(1+c) a\left(1+c+c^{2}\right)$ but the three components of this language 
are not disjoint, since they all contain $a a$. Note that the words $a c a b, a b a c, b a c a$ and caba are not in $K \cap L$.

The syntactic ordered monoid of $K_{0}$ and $L_{1}$ has 4 elements $\{1, a, b, c\}$ and is presented by the relations $a=b a=b^{2}=b c=c a=c b=0$ and $c^{2}=b$. Its syntactic order is defined by $a<b<c<1$.

The syntactic ordered monoid of $L_{0}$ and $K_{1}$ has 13 elements:

$$
\left\{1, a, b, c, a^{2}, a b, a c, b a, c a, c^{2}, a c^{2}, b a b, c a c\right\}
$$

and is defined by the relations $c a c^{2}=b a b$ and

$$
b^{2}=b c=c b=a^{2}=a b a=a c a=b a c=c a b=c^{2} a=c^{3}=0 .
$$

The syntactic order is:

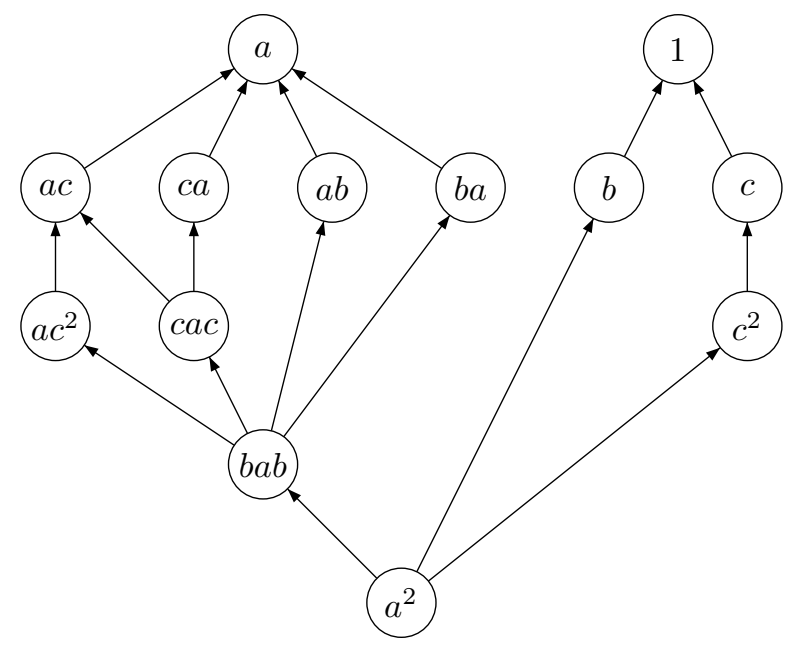

There is only one pair of infiltration maps $(\alpha, \beta)$ of $I(1,1)$ that defines a nonempty set $U(\alpha, \beta)$. This pair is defined as follows: $\alpha(1)=1$ and $\beta(1)=2$. The triples $\left(z_{0}, z_{1}, z_{2}\right)$ of $C(\alpha, \beta)$ are exactly the triples of words such that $z_{0} a z_{1} a z_{2} \in K \cap L$. In particular, $z_{0} \in\{1, b, c\}, z_{1} \in\{1, b, c\}$ and $z_{2} \in\left\{1, b, c, c^{2}\right\}$. Now, one has

$$
\begin{array}{llll}
{\left[K_{0}\right]_{\uparrow 1}=1} & {\left[K_{0}\right]_{\uparrow b}=1+b+c+c^{2}} & {\left[K_{0}\right]_{\uparrow c}=1+c} & \\
{\left[K_{1}\right]_{\uparrow 1}=1} & {\left[K_{1}\right]_{\uparrow b}=1+b} & {\left[K_{1}\right]_{\uparrow c}=1+c} & {\left[K_{1}\right]_{\uparrow c^{2}}=1+c+c^{2}} \\
{\left[L_{0}\right]_{\uparrow 1}=1} & {\left[L_{0}\right]_{\uparrow b}=1+b} & {\left[L_{0}\right]_{\uparrow c}=1+c} & \\
{\left[L_{1}\right]_{\uparrow 1}=1} & {\left[L_{1}\right]_{\uparrow b}=1+b+c+c^{2}} & {\left[L_{1}\right]_{\uparrow c}=1+c} & {\left[L_{1}\right]_{\uparrow c^{2}}=1+b+c+c^{2}}
\end{array}
$$


which gives the following possibilities for the triples $\left(U_{0}, U_{1}, U_{2}\right)$, for the following triples $z=\left(z_{0}, z_{1}, z_{2}\right)$ :

$$
\begin{array}{llll}
z=(1,1,1) & U_{0}=1 & U_{1}=1 & U_{2}=1 \\
z=(b, b, b) & U_{0}=1+b & U_{1}=1+b & U_{2}=1+b \\
z=(c, c, c) & U_{0}=1+c & U_{1}=1+c & U_{2}=1+c \\
z=\left(b, c, c^{2}\right) & U_{0}=1+b & U_{1}=1+c & U_{2}=1+c+c^{2} \\
z=\left(c, c, c^{2}\right) & U_{0}=1+c & U_{1}=1+c & U_{2}=1+c+c^{2}
\end{array}
$$

\subsection{Deterministic product}

The marked product $L=L_{0} a_{1} L_{1} \cdots a_{n} L_{n}$ of $n$ nonempty languages $L_{0}, L_{1}$, $\ldots, L_{n}$ of $A^{*}$ is left deterministic [right deterministic] if, for $1 \leqslant i \leqslant n$, the set $L_{0} a_{1} L_{1} \cdots L_{i-1} a_{i}\left[a_{i} L_{i} \cdots a_{n} L_{n}\right]$ is a prefix [suffix] code. This means that every word of $L$ has a unique prefix [suffix] in $L_{0} a_{1} L_{1} \cdots L_{i-1} a_{i}\left[a_{i} L_{i} \cdots a_{n} L_{n}\right]$. It is observed in [3, p. 495] that the marked product $L_{0} a_{1} L_{1} \cdots a_{n} L_{n}$ is deterministic if and only if, for $1 \leqslant i \leqslant n$, the language $L_{i-1} a_{i}$ is a prefix code. Since the product of two prefix codes is a prefix code, we get the following proposition.

Proposition 5.11. Any left [right $]$ deterministic product of left [right $]$ deterministic products is left [right] deterministic.

Proof. This follows immediately from the fact that the product of two prefix codes is a prefix code.

Factorizing a deterministic product also gives a deterministic product. More precisely, one has the following result.

Proposition 5.12. Let $L_{0} a_{1} L_{1} \cdots a_{n} L_{n}$ be a left [right] deterministic product and let $i_{1}, \ldots, i_{k}$ be a sequence of integers satisfying $0<i_{1}<\ldots<i_{k}<n$. Finally, let $R_{0}=L_{0} a_{1} L_{1} \cdots a_{i_{1}-1} L_{i_{1}-1}, \ldots, R_{k}=L_{i_{k}} a_{i_{k}+1} L_{i_{k}+1} \cdots L_{n-1} a_{n} L_{n}$. Then the product $R_{0} a_{i_{1}} R_{1} \cdots a_{i_{k}} R_{k}$ is left [right] deterministic.

Proof. Trivial.

The left [right] deterministic polynomial closure of a class of languages $\mathcal{L}$ of $A^{*}$ is the set of languages that are finite unions of left [right] deterministic products of the form $L_{0} a_{1} L_{1} \cdots a_{n} L_{n}$, where the $a_{i}$ 's are letters and the $L_{i}$ 's are elements of $\mathcal{L}$.

We can now state the counterpart of Theorem 5.3 for deterministic products.

Theorem 5.13. If the products $K=K_{0} a_{1} K_{1} \cdots a_{p} K_{p}$ and $L=L_{0} b_{1} L_{1} \cdots b_{q} L_{q}$ are deterministic, the products occurring in Formula (4) are all deterministic.

Proof. Let $i \in\{0, \ldots, r\}$. By construction, there exists $k \geqslant 0$ such that $i+1=$ $\alpha(k+1)$ or $i+1=\beta(k+1)$. By Lemma 4.2, there exists $j \leqslant i$ such that either $U_{j} c_{j+1} U_{j+1} \cdots U_{i} \subseteq K_{k}$ and $c_{\alpha(k+1)}=a_{k+1}$ or $U_{j} c_{j+1} U_{j+1} \cdots U_{i} \subseteq L_{k}$ and 
$c_{\alpha(k+1)}=b_{k+1}$. Suppose we are in the first case and that $U_{i} c_{i+1}$ is not a prefix code. Then $U_{j} c_{j+1} U_{j+1} \cdots U_{i} c_{i+1}$ is not a prefix code and thus $K_{k} a_{k+1}$ is not a prefix code. This yields a contradiction since the product $K_{0} a_{1} K_{1} \cdots a_{p} K_{p}$ is deterministic.

Corollary 5.14. Let $\mathcal{L}$ be a lattice of regular languages closed under quotient. Then its deterministic polynomial closure is also closed under quotient.

\section{Acknowledgements}

I would like to thank Mário J. J. Branco for his careful reading of a first version of this article.

\section{References}

1. M. Arfi, Opérations polynomiales et hiérarchies de concaténation, Theoret. Comput. Sci. 91 (1991), 71-84.

2. M. J. J. Branco, On the Pin-Thérien expansion of idempotent monoids, Semigroup Forum 49,3 (1994), 329-334.

3. M. J. J. BRANCO, The kernel category and variants of the concatenation product, Internat. J. Algebra Comput. 7,4 (1997), 487-509.

4. M. J. J. BRANCO, Two algebraic approaches to variants of the concatenation product, Theoret. Comput. Sci. 369,1-3 (2006), 406-426.

5. M. J. J. BRANCO, Deterministic concatenation product of languages recognized by finite idempotent monoids, Semigroup Forum 74,3 (2007), 379-409.

6. M. J. J. BRANCO AND J.-E. PIN, Equations for the polynomial closure, in $I C A L P$ 2009, Part II, S. Albers and W. Thomas (eds.), pp. 115-126, Lect. Notes Comp. Sci. vol. 5556, Springer, Berlin, 2009.

7. M. Lothaire, Combinatorics on words, Cambridge Mathematical Library, Cambridge University Press, Cambridge, 1997.

8. D. Perrin And J.-E. Pin, Infinite Words, Pure and Applied Mathematics vol. 141, Elsevier, 2004. ISBN 0-12-532111-2.

9. J.-E. PIN, Propriétés syntactiques du produit non ambigu, in 7th ICALP, pp. 483499, Lect. Notes Comp. Sci. n 85, Springer, Berlin, 1980.

10. J.-E. PIN, A variety theorem without complementation, Russian Mathematics (Iz. VUZ) 39 (1995), 80-90.

11. J.-E. Pin, H. Straubing And D. Thérien, Locally trivial categories and unambiguous concatenation, J. of Pure and Applied Algebra 52 (1988), 297-311.

12. J.-E. Pin AND D. ThÉRIEn, The bideterministic concatenation product, Internat. J. Algebra Comput. 3 (1993), 535-555.

13. J.-E. PIN AND P. WeIL, Polynomial closure and unambiguous product, Theory Comput. Systems 30 (1997), 1-39.

14. M.-P. Schützenberger, Une théorie algébrique du codage, in Séminaire DubreilPisot, année 1955-56, Exposé No. 15, 27 février 1956, 24 pages, Inst. H. Poincaré, Paris, 1956.

15. M.-P. Schützenberger, Sur le produit de concaténation non ambigu, Semigroup Forum 18 (1976), 331-340. 